\title{
La genèse en chanson : constantes et spécificités
}

\section{Stéphane Chaudier et Joël July}

\section{(2) OpenEdition}

\section{Journals}

Édition électronique

URL : https://journals.openedition.org/genesis/5779

DOI : 10.4000/genesis. 5779

ISSN : 2268-1590

\section{Éditeur :}

Presses universitaires de Paris Sorbonne (PUPS), Société internationale de génétique artistique littéraire et scientifique (SIGALES)

\section{Édition imprimée}

Date de publication : 1 juillet 2021

Pagination : 7-18

ISBN : 979-10-231-0710-4

ISSN : 1167-5101

\section{Référence électronique}

Stéphane Chaudier et Joël July, «La genèse en chanson : constantes et spécificités », Genesis [En ligne], 52 | 2021, mis en ligne le 01 juillet 2021, consulté le 17 août 2022. URL : http:// journals.openedition.org/genesis/5779; DOI : https://doi.org/10.4000/genesis.5779 


\title{
La genèse en chanson : constantes et spécificités
}

\author{
Stéphane Chaudier et Joël July
}

$L$ a chanson est-elle un bon candidat pour les études génétiques? Pionnière des analyses universitaires sur la chanson, Ursula Mathis-Moser rappelle ici même, dans son article sur Le Déserteur de Vian, une distinction utile : la promotion du «manuscrit littéraire comme objet scientifique» serait la tâche principale de la génétique; l'étude générale des «processus de la création dans la littérature, les arts et les sciences», beaucoup plus vaste, fait appel à des considérations psychologiques, biographiques ou sociohistoriques, qui excèdent la prise en compte des brouillons. Dans lequel de ces deux champs inscrire l'étude de la chanson? Faut-il en exclure un, privilégier l'autre?

Les mots genèse et création sont synonymes; tous les deux désignent un processus qui appelle un récit. Raconter et expliquer la genèse d'une æuvre, c'est dire comment elle a été produite, c'est en faire la biographie. Ce récit est scientifique quand il présente un certain nombre de critères de rationalité; les étapes de la création y sont attestées par des documents datables et datés; c'est le travail de la génétique. Quand un auteur, un parolier, un compositeur, un interprète rapporte son propre récit de création ou genèse chansonnière, il livre un témoignage sans prétention à la scientificité, lequel appelle l'esprit critique. Ce numéro s'appuiera sur ces deux modalités discursives (le récit génétique savant et le récit génétique intuitif) pour tenter de répondre à ces questions clés: le processus de création en chanson obéit-il à des constantes? Présente-t-il des spécificités?

\section{La chanson : l'air et les paroles}

Commençons par dire ce qui fâche. La chanson est, à l'évidence, un parent pauvre des études littéraires ${ }^{1}$. Populaire, honteuse et donc longtemps anonyme - le phénomène des chansons signées est tout récent ${ }^{2}-$, la chanson est-elle même à proprement parler un texte

1. Qu'entendre par chanson? Chez les spécialistes, un consensus se dessine : la chanson est un air (ou une mélodie) associé à des paroles. La spécificité de la chanson française tient à l'importance des paroles : le texte y est chanté pour être compris et repris par les auditeurs. Ce souci de la mémorisation favorise le recours et le maintien (aujourd'hui majoritaire mais non exclusif) du vers, de la rime et de la strophe. Ces contraintes sont certes adaptées aux exigences d'un genre populaire (rimes de mirliton, numérisme approximatif du vers, système strophique dominé par l'alternance refrain / couplet); elles sont remises en cause par des genres ou des artistes modernistes (pratique du vers libre, du texte en prose poétique récité, talk over, rap) et/ou remotivées par des artistes littéraires. Voir à ce sujet Joël July, «Le vers de chanson, de la convergence à la divergence», Europe, Poésie et chanson, mars 2020, p. 120-142 et Stéphane Chaudier, «Le grêle et le vivace aujourd'hui : l'effet rime en chanson», ibid., p. 100-119; des mêmes auteurs, «L'étoffe de la strophe en chanson», dans M. Monte et al. (dir.) Stylistique et Méthode, Presses universitaires de Lyon, 2018, p. 345-360.

2. Voir Jean-Nicolas de Surmont, Vers une théorie des objets-chansons, Lyon, ENS éditions, 2011. L'objet-chanson, fixé, parfois signé et donc assumé comme œuvre par des artistes ou des créateurs, s'oppose à la pratique chansonnière comme phénomène anthropologique, historique, socioculturel. 
littéraire 3 ? Le fait même qu'il y ait des chansons à texte montre bien qu'il existe, a contrario, des chansons sans texte, c'est-à-dire dont les paroles ne valent rien ${ }^{4}$. Mais Bertrand Belin va beaucoup plus loin que ce simple constat; dans l'entretien mené par Gaspard Turin, il interroge la fausse évidence de ce qu'on pourrait appeler la littérarité ou la textualité de la chanson:

La destination des chansons est les oreilles, et par ailleurs je ne pense pas qu'il faille que leurs textes soient disponibles à la lecture. Si je les publie dans la pochette de l'album, c'est que mon label m'enjoint de le faire. Le public en général aime bien ça. Mais moi, qui ne les ai pas écrits, n'ayant fait que les enregistrer, j’ai de la peine à passer par ce stade !

Si la chanson se fait avec des mots, ces mots ne sont pas réductibles à leur trace écrite, puisqu'ils interagissent avec une voix, des instruments et les données sensibles d'un spectacle 5 : "J'essaie donc de passer de la voix, de l'esprit presque, à l'enregistrement directement, comme si l'enregistrement captait les fantômes», ajoute Bertrand Belin, qui avoue volontiers faire l'économie de la page. Une métaphore saisissante semble remettre en cause l'objet de ce dossier:

[...] petit à petit, j'en suis arrivé à ce constat que la musique est le biotope des mots de la chanson.

C'est dans ce biotope que les mots agissent et sont vivants, comme des poissons dans un aquarium.

Ils vont pouvoir vivre dans la chanson. Si je les en sors pour les mettre sur une page, ils vont crever.

Attention, cimetière en vue! La chanson serait non seulement bien plus qu'un texte (vérité accessible à quiconque a des yeux et surtout des "oreilles», comme le dit si bien Bertrand Belin) mais aussi tout autre chose qu'un texte. Ce charme et cette spécificité de la chanson (art pluri-sémiotique, convoquant la musique, les arts de la scène et même les arts visuels, quand elle interagit avec un clip) ne la qualifieraient nullement pour être un texte et surtout pas un texte littéraire. L'argument vaut la peine d'être entendu. Malgré tout, les responsables de ce dossier, stylisticiens de formation, estiment qu'on peut étudier les caractéristiques langagières du genre chanson sans le trahir. Deux conceptions s'opposent à ce sujet. L'une, organiciste et synthétiste, soutient que la chanson est un tout en interaction constante (texte, voix, instrument, scène, images de clip, etc.) et que la réception d'une telle ouvre est le fait d'un corps-esprit centralisateur, sommé par le genre chanson d'articuler ces différentes composantes. L'autre,

3. Qu'entendre par littéraire? Un texte littéraire (produit ou reconnu comme tel) appelle la relecture studieuse ou savante; il suscite le conflit des interprétations, en raison de la pluralité de ses sens, de la complexité de ses élaborations formelles. En revanche, un texte non littéraire comme une chanson commerciale serait un texte sur lequel le spécialiste de littérature ne trouve rien à dire : la mise en relation du fond et des formes n'y est pas selon lui assez problématique. La littérarité d'une chanson (ou sa poéticité) est à l'évidence un critère à la fois labile et décisif : il favorise la perception de sa valeur; il décide souvent (mais non systématiquement) de son inscription dans le patrimoine chansonnier.

4. Le célèbre Boum! de Charles Trenet (1938) montre qu'un propos superficiel ne nuit pas toujours à la qualité ni au succès d'une œuvre : car les mots, véritable «chair à chanson», sont aussi (voire avant tout) un tremplin pour la mélodie, la virtuosité vocale, d'où les mélismes (plusieurs notes portant une syllabe) ou l'emploi si fréquent des onomatopées.

5. C'est à Stéphane Hirschi que revient le mérite d'avoir pris appui sur cette évidence pour fonder une discipline ambitieuse nommée cantologie. Dans cette vaste demeure, comme dans celle du Père, plusieurs chapelles coexistent pacifiquement. Stéphane Hirschi appelle de ses vœux une sémiologie inclusive, qui prenne en compte tous les aspects de la chanson. 
la nôtre, est analytique; elle déconstruit cette organicité idéalisée au nom de l'empirisme. La chanson est faite de divers modules sémiotiques plus ou moins autonomes, plus ou moins saillants, et dont l'articulation est non prescriptive : on peut aimer Brassens sans aimer la guitare et donc fermer partiellement ses oreilles à l'écoute de L'Auvergnat; inversement, on peut aimer Brassens sans comprendre le français ${ }^{6}$. La chanson est faite pour mettre en valeur des institutions culturelles et historicisées (la voix humaine, la musique, la langue, la textualité, la scène) aussi bien que des appropriations singulières. Écouter et aimer Brassens, c'est donc percevoir (simultanément ou diversement) et admirer (plus ou moins) une voix, une langue (la langue française), une écriture (poétique), un instrument, une posture, une présence corporelle et scénique, autant dire un rapport contextualisé à la voix, à la langue, au style, à la scène, à l'espace public; mais rien n'oblige l'amateur ou le spécialiste à prendre en charge l'intégralité de ce qui serait un paquetage identitaire générique. L'organicisme chansonnier est donc un idéalisme. L'expérience montre que l'articulation des divers modules de la chanson est à la fois circonstanciée, non impérative et jamais pleinement réalisée; elle ne saurait être un préalable épistémologique à l'étude (nécessairement plurielle et sectorielle) du genre chanson. Dans la vie de tous les jours (même si, de moins en moins, on rencontre la situation qu'évoque ce simple et splendide vers d'Aragon: "Comme au passant qui chante on reprend sa chanson ${ }^{7}$ »), on siffle et on retient l'air d'une chanson pour une foule de raisons où la poésie n'occupe que rarement la première place; en revanche, c'est par l'entremise et la grâce de leurs paroles que les chansons s'inscrivent dans la mémoire collective puis dans le panthéon national 8 . Oui, mais comment? La génétique peut-elle nous aider à y voir plus clair?

Comme le théâtre, la chanson connaît parfois un accouchement multiple : entre la création, le dépôt à la SACEM (depuis 1851) et la profération sur scène, quel texte faut-il prendre en compte? Bien des artistes depuis Brel rodent leurs chansons dans des récitals sans

6. Voir l'article de Jacopo Tomatis, «L'enfant et le malentendu», J. July et C. Chabot-Canet (coord.), Du malentendu dans la chanson, Aix-en-Provence, PUP, coll. «Chants Sons», 2021, p. 181-192.

7. «Que serai-je sans toi»; ces vers d'Aragon, extraits de «Prose du bonheur et d'Elsa», long poème qui conclut Le Roman inachevé (1956), ont été mis en voix par Ferré, par Ferrat, puis bien d'autres.

8. C'est tout l'objet d'une stylistique de la chanson de montrer comment l'auteur (ou le parolier) montre et magnifie divers états de la langue française. Brassens et Barbara (celle-ci jusqu'en 1973) célèbrent une langue poétique, faisant bon accueil au haut registre et à une versification que d'aucuns peuvent juger surannée; Gainsbourg fait chatoyer un français métissé d'anglais; Renaud invente et accrédite une langue argotique contemporaine (devenue représentative des quartiers populaires dits sensibles); élue pour représenter la France à l'Eurovision 2021 avec sa chanson Voilà, Barbara Pravi choisit de mettre en valeur les ressources qu' offre une langue courante à l'expression du lyrisme et du pathos. On parle de patrons (Gilles Philippe) quand un faisceau stabilisé de traits repérables et repérés comme tels produisent un effet identique (il existe par exemple un patron grivois, fondé sur une scénarisation caractéristique, un stock lexical, un art de l'hyperbole, du sous-entendu, du jeu de mots, etc.); on parle de style quand un auteur impose une manière singulière de combiner et ou de transformer des patrons mis à disposition de tous. Joël July a forgé le terme populariste pour désigner le fait que la chanson, genre populaire (qui cherche le succès du plus grand nombre), est fondée sur l'usage et la réélaboration littéraire de formes connues de tous (et non seulement de l'élite) : $1^{\circ}$ la langue parlée (l'oral, et en particulier celui des jeunes); $2^{\circ}$ les codes éprouvés (le vers, pour faire retenir ce qui touche et qui plaît). Le défigement des expressions figées, l'usage expressif de syntaxèmes du français familier, la polyphonie, les échos phoniques sont les traits majeurs de cette esthétique qui veut allier la simplicité et la complexité. L'esthétique populariste est donc à la fois un conservatoire (par exemple du vers et du refrain) et un laboratoire (elle valorise le français qui se parle, son lexique et sa syntaxe). Cette esthétique transcende tous les patrons et tous les styles; elle définit le style du genre chanson française. Voir Joël July, Esthétique de la chanson française, Paris, L'Harmattan, 2007. 
les avoir préalablement fixées ni définitivement enregistrées sur un support discographique. Le texte en chanson est une réalité flottante. Joël July, à propos du cas exemplaire de Barbara, montre que les «variantes » ne sont pas toutes volontaires ${ }^{9}$ : elles tiennent à la mise en voix, où jouent aussi bien des hasards de la mémorisation que des trouvailles impromptues qu'un enregistrement pérennise. Olivier Migliore, travaillant sur deux interprétations d'un texte de Moustaki (celle de Reggiani puis de Moustaki lui-même), prouve combien la signification, l'effet et les émotions d'une chanson varient d'une "performance » à l'autre. À l'inverse, notre génération a peut-être moins conscience de cette fluidité inhérente au texte de la chanson, mouvant au gré des interprétations vocales : l'émission de Nagui, N'oubliez pas les paroles!, repose sur l'idée, certes discutable mais bien ancrée dans les consciences, qu'une chanson est un produit fini et inviolable (comme l'est toute ouvre d'art prestigieuse 10); le but du jeu (puisque c'en est un...) est de lui appliquer le principe de la récitation scolaire, si bien que des candidats méritants peuvent échouer pour une simple erreur sur un déterminant ou une conjonction. Mais cette émission et le succès qu'elle connaît traduisent-ils l'adhésion du public à cette représentation nouvelle de l'objet-chanson? La chanson, ouvre d'art populaire, devient le prétexte de la virtuosité mémorielle; et ce jeu télévisé finit sans doute par refléter banalement l'amour (si contemporain!) de la compétition féroce et de ses règles arbitraires... Il n'en demeure pas moins qu'il entre dans un processus de relative sanctuarisation des paroles de ces auvres autrefois considérées comme futiles que sont les objets-chansons et il vient donc légitimer notre travail de prise en compte scrupuleux des mouvements des textes. Pas de plus grandes difficultés pour les candidats que ceux qui contiennent des listes ou des refrains variants ou des calembours improbables et loufoques. Cette attention aux mots de la chanson s'oppose à la frivolité à laquelle on les réduit parfois en les remplaçant par des lallations; elle justifie le travail de fond que les auteurs de ce numéro ont mené.

\section{Spécificités du genre chanson}

La section «Enjeux» ouvre le dossier. L'article liminaire de Stéphane Chaudier pointe deux spécificités chansonnières qui compliquent singulièrement la tâche du généticien. D'une part, les producteurs de chansons (Auteurs Compositeurs Interprètes, désormais ACI, et musiciens, pour s'en tenir aux premiers rôles ${ }^{11}$ ) prétendent souvent travailler vite, et surtout de manière non scolaire : affabulation ou réalité? Posture ou méthode? Telle ou telle chanson aurait été écrite sur un coin de table (puisque le genre est à la fois bref et mineur, selon une doxa qu'on peut estimer désinvolte ou réaliste); elle se serait imposée sous l'emprise généreuse de l'excitation-inspiration produite par l'alcool, les stupéfiants, la sociabilité artiste, la pression des contraintes professionnelles, pour ne rien dire des vertus du hasard.

\footnotetext{
9. Une variante qu'on peut ramener à une intention d'auteur est indiscutablement significative; si elle tient au hasard, la preuve de la significativité repose tout entière sur les épaules de l'interprète.

10. Dans la musique, les théoriciens opposent les tenants d'une théorie scripturaliste, pour lesquels c'est la partition, texte musical, qui fait l'identité de l'œuvre, et ceux qui valorisent la performance musicale, changeante.

11. De ce que tous ces mots sont employés au genre du masculin, selon la convention en vigueur, il ne s'ensuit nullement que les auteurs de ce collectif minorent, ignorent et encore moins méprisent la contribution des femmes à la chanson.
} 
Beaucoup de chansons métachansonnières qui s'érigent en art poétique évoquent au contraire les tâtonnements de l'artiste : Pour essayer de faire une chanson (Aznavour), J'écris faux, je chante de la main gauche (Doremus), J'aime pas la chanson de Juliette (dont le titre constitue un habile trompe-l'ail, dissimulant un éloge)12. En chanson, le processus génétique ne conserve pas forcément de trace manuscrite ou tapuscrite : parce qu'il ambitionne d'être populaire (aimé et connu de tous, sans distinction de classes sociales ou de culture), l'artiste y est moins qu'ailleurs considéré comme un intellectuel, et davantage comme une vedette, un personnage public surmené13. Pour lui, le travail se fait souvent dans la boîte noire de l'inaccessible intériorité. La rumination d'une chanson peut prendre des années : soit; mais en l'absence de documents qui attestent cette lente élaboration, comment en décrire les phases autrement qu'en se fiant à la parole du témoin, l'artiste ou ses proches? Bertrand Belin, dans l'entretien avec Gaspard Turin, ne dit pas autre chose... Mais encore faut-il comprendre les raisons d'un tel diagnostic et pourquoi ce dernier n'invalide nullement la perspective génétique ${ }^{14}$.

Depuis 1955, le statut du chanteur est reconnu par la mise en place de droits spécifiques reversés à l'interprète par le biais de l'Adami (Administration des droits des artistes et musiciens interprètes). Pour autant, la question de la propriété intellectuelle, si elle existe, n'est pas vécue sur le même mode qu'en littérature. La notion de plagiat musical est reléguée derrière une palette d'influences et d'inspirations plus ou moins avouées ${ }^{15}$; d'une langue à l'autre, sous la pratique du cover, les textes de chanson circulent, s'adaptent à leur public, se déforment, sans que nul n'y trouve à redire. Surtout, l'intérêt commercial bien compris et le compagnonnage artistique facilitent les emprunts des uns au répertoire des autres. Cora Vaucaire interpréta la première Dis, quand reviendras-tu? avant son auteure, Barbara16. Mouloudji l'interpréta lui aussi, un peu plus tard, en faisant varier le vers pour l'adapter à son sexe (ou à son genre) : "Je n'ai pas la vertu des femmes de marin » devient ainsi

12. Nous prenons parti en faveur des titres de chanson traités typographiquement en italiques comme ceux d'œuvre à part entière. L'arrivée des chanteurs poètes dans les années cinquante et la constitution en album (33-T puis CD dix-douze pistes) de l'après-guerre aux années 1990 ont favorisé une réception de la chanson comme pièce d'un ensemble, à l'instar d'un poème : le titre pouvait y sembler une citation et favoriser ainsi l'emploi des guillemets. C'est une réalité caduque pour la chanson qui précède (78-T ou petites formes) et celle qui existe aujourd'hui (CD single, streaming), non avenue pour sa réception à la radio, à la télévision, sur Internet et en concert où se succèdent des pièces issues d'albums différents de l'artiste, des créations et des reprises. La chanson, dans sa réception moderne, a donc un statut différent du poème qui doit nous inciter à la traiter, isolément et comme un tout malgré sa brièveté, d'où l'emploi de l'italique pour son titre.

13. Un ACI (ou ses ayants droit) confie rarement ses archives à un institut spécialisé comme l'IMEC; dans l'inventaire en ligne des collections de cette institution, le mot chanson n'apparaît pas, ni sous la rubrique «Littérature », ni sous la rubrique «Arts », laquelle spécifie pourtant : «arts de la scène», «arts plastiques », « cinéma », «musique». Les fameux trois B (Brel, Barbara, Brassens - à l'origine Brassens, Brel, Béart), ou Gainsbourg, pour s'en tenir aux monstres sacrés de la chanson française, ne figurent pas dans cet inventaire.

14. Comme on le verra, la genèse d'une chanson se situe autant avant qu'après la première énonciation publique. Ainsi s'explique en partie le faible prestige de l'archive pré-éditoriale (ou pré-communicationnelle) en chanson; elle valide la méthodologie adoptée par plusieurs contributions du numéro : celle d'une génétique post-éditoriale (post-communicationnelle), qui opère par comparaison de versions chronologiquement organisées et qui cherche à décrire les conditions de la création d'une œuvre collaborative et déjà publique. (Note de Rudolf Mahrer.)

15. Voir Michaël Andrieu, Réinvestir la musique, Paris, L'Harmattan, coll. «Univers musical», 2011.

16. Pour les conditions de ces versions concomitantes, lire Gilles Schlesser, Le Paris de Barbara, Paris, Parigramme, 2019 , p. 36. 
«Je n'ai pas la vertu des chevaliers anciens». Ce même Mouloudji interprète Le Déserteur concomitamment à Boris Vian (voir l'article d'Ursula Mathis-Moser, p. 79), comme Georges Brassens ${ }^{17}$ ou comme Juliette Gréco ou Serge Reggiani dans ces mêmes années. Dans les années soixante, on se rend volontiers hommage en s'empruntant des chansons. Georges Moustaki, ACI, chanta Ma liberté après la version initiale de Serge Reggiani (voir l'article d'Olivier Migliore, p. 95). Il n'est donc pas rare que le créateur ne soit pas à l'initiative de la version la plus ancienne. C'est encore le cas aujourd'hui : Carla Bruni, auteure de la chanson Déranger les pierres, en enregistre une version après que Julien Clerc, son compositeur, l'a interprétée ; ayant écrit Le Garçon triste, elle le laisse à Isabelle Boulay (album En vérité, 2017), avant de le reprendre elle-même sur son propre album Carla, en 2020.

Dans un long article sur les reprises ${ }^{18}$, Joël July a isolé des situations particulières où la version d'un nouvel interprète ne présente pas seulement un écart temporel par rapport au texte source; elle manifeste aussi des variations performancielles qui réorientent considérablement les intentions et la perception originelles. La «montée en gamme esthétique »'une chanson comme Les Mots bleus (de la bluette sentimentale de Christophe à sa recréation par Bashung) ou à l'inverse, le passage d'une chanson d'artiste plutôt confidentielle à une chanson pop réussie (Mister Mystère écrite par Brigitte Fontaine pour elle-même et reprise par M, le compositeur, qui la transforme en succès populaire) suscite la perplexité bien tempérée du spécialiste : malgré l'identité du titre, a-t-on affaire, oui ou non, à la même auvre19? La reprise vaut comme processus de recatégorisation, affirme Joël July. Certes, la chanson reprise est modifiée, c'est l'évidence même, par la différence des pratiques vocales et des facteurs musicaux ${ }^{20}$; mais le sentiment que la nouvelle version n'est plus tout à fait, voire plus du tout, la même chanson que "l'originale» s'accroît en raison des interférences entre le statut de l'interprète et le je du canteur (ou de la cantrice). En reprenant un titre auquel le premier interprète donnait une certaine orientation, l'artiste qui lui succède infuse quelque chose de son univers, de son style, de sa réputation; son propre répertoire chansonnier, l'image publique qu'il s'est constituée, les marques de son état civil (sexe, âge, physique) modifient la perception de la chanson. L'ethos intradiscursif du canteur - réputé ne pas varier au moment d'une reprise à l'identique - est ainsi contaminé, remodelé, par un ethos extradiscursif plus prégnant : celui de l'interprète.

Exagération? On en jugera par un exemple concret, en confrontant Les Filles du bord de mer dans la version originale de Salvatore Adamo (version de moins de trois minutes dont

17. En ligne sur youtube.com.

18. Joël July, «En quoi la reprise est une réécriture... », dans P. Abbrugiati (dir.), Réécriture et chanson dans l'aire romane, Presses universitaires de Provence, coll. «Chants Sons», 2017, p. 85-118. Cette proposition théorique tend à infléchir la conception institutionnelle de la chanson par le biais d'un phénomène qui en atteste la vitalité : la reprise. Véritable réinterprétation de la chanson initiale, celle-ci peut être reçue comme réécriture.

19. Pour les auditeurs des années soixante-dix, Je vole est indiscutablement une chanson de Michel Sardou; pour la jeune génération, depuis 2014, c'est une chanson de Louane. Le de à droite d'un titre de chanson introduit le plus souvent le nom de la vedette grâce à laquelle la chanson atteint le public; cette simple observation suffit à montrer à quel point le genre chansonnier relativise, voire périme, les approches trop strictement «auteuristes » de l'œuvre d'art - ce qui ne manque pas d'influer sur l'approche génétique de la chanson.

20. Voir Céline Chabot-Canet, Interprétation, phrasé et rhétorique vocale dans la chanson française depuis 1950 : expliciter l'indicible de la voix, thèse, université Louis Lumière - Lyon II, 2013, en ligne sur tel.archives-ouvertes. fr. Voir notamment p. 557 et p. 798 pour les différentes interprétations des Feuilles mortes. 
il est l'ACI21) à celle d'Arno (version de près de cinq minutes 22 ). Le jeune Adamo, belge d'origine sicilienne, surfant sur la vague yéyé, doit son succès à son image de gendre idéal qui a interprété Vous permettez, Monsieur. Rien de tel, dans cette chanson humoristique; elle offre une satire des midinettes hantant les plages $d u$ Nord, peufarouches 23 et soucieuses de leur ligne avant tout; mais elles ne sont pourtant pas plus ridicules que ce canteur naïf et vulgaire, séducteur peu doué, dupé et pas content de l'être, véritable tête de turc de son propre récit : les «oin oin» à la fin des refrains, la contraction familière ( z'étaient chouettes »), les [r] parodiquement roulés... Le rythme en valse musette ressemble à une java populaire des bords de Marne : le texte y fait d'ailleurs référence ( $Z$ 'auraient pu danser la java»). Le refrain «Z'étaient chouettes les filles du bord de mer / Z'étaient faites pour qui savait y faire » ne peut nous faire douter de l'intention franchement comique de la chanson. Trente ans plus tard, son compatriote Arno, ACI lui-même, propose une interprétation diamétralement différente; un accordéon larmoyant accompagne la voix très rocailleuse, traînante, aux accents flamands, cohérente avec cet ethos extradiscursif d'artiste fiévreux, rebelle, élitiste, qui caractérise Arno. Les « oin oin» de Salvatore Adamo sont remplacés par des «chouette chouette » éructés, la liaison ridicule ( $z$ 'étaient») fait place à un démonstratif moins caricaturalement populaire : "c'était chouette». C'est à une tragédie quasi brélienne que ce texte nous convie : un homme revient sur la trahison féminine avec une amertume que les refrains soulignent au lieu de l'exorciser par le rire partagé. L'anecdote n'est plus un détail dans un parcours de vie : le pluriel («les filles») qui permettait la satire chez Adamo atteste chez. Arno l'indéniable fatalité des amours déçues. Quand la reprise ménage un écart tel entre les prestations vocales, quand la volonté de décalage est si assurée, si assumée, alors même que le texte n'est que très marginalement modifié, la réécriture est avérée: la divergence des ethê fait qu'il n'y a plus une chanson et sa reprise, mais une création continuée, d'une interprétation à l'autre, dont la première est le support mais non le modèle de l'autre.

Outre le statut plastique du texte de chanson, la genèse de ce genre si particulier ne peut pas ignorer la dimension collective du travail créatif 24 . On est ainsi conduit à se demander quel est le lieu privilégié de la genèse en chanson et quel est son support le plus typique. Le bureau, la feuille de papier ou le fichier d'ordinateur? Ou bien le studio d'enregistrement, avec les instruments de musique et la console de l'ingé-son? Sous le nom prestigieux, syncrétiste, fédérateur et quelque peu fallacieux de Julien Clerc ou de Johnny Hallyday, il y a peut-être autant de "styles de chanson» qu'ils ont eu d'auteurs et de compositeurs : usuellement, on rattache la genèse d'une ouvre littéraire à un nom d'auteur; ce qui, en chanson, on l'a vu, est bien problématique. À qui attribuer telle ou telle trouvaille langagière, par laquelle rétrospectivement on expliquera la beauté et le succès de la chanson? Au parolier? À l'interprète prescripteur ou relecteur? Au directeur artistique? Faut-il, dans nos analyses, prendre en compte le rôle ou la pression du manager, de l'éditeur,

21. Salvatore Adamo, 45-T, 1964 (EPG, La Voix de son maître), Adamo a 21 ans. En ligne sur youtube.com. 22. Arno, album Idiots savants, 1993, Arno a 44 ans. En ligne sur youtube.com.

23. «Elle invitait le premier baigneur / À tâter du côté de son cœur / En douceur, en douceur / En douceur et profondeur. » L'obscénité prend à peine le soin de se gazer.

24. Voir Joël July, «Singulièrement pluriel», dans J. July (dir.), Chanson. Du collectif à l’intime, Aix-en-Provence, PUP, coll. «Chants Sons », 2016, p. 7 à 24. Et la note 14, supra. 
du producteur, de la star, et le cahier des charges qu'ils assignent au créateur? Boris Vian répondrait que oui :

Quand vous avez dans le texte remplacé «à poil» par «tout nu» et «faire l'amour»par «parler d'amour», quand, au lieu de partir avec le magasinier, le héros pédéraste de votre chanson s'envole avec la dactylo (ce qui fausse entièrement le sens de votre démonstration), quand le compositeur s'est fait agonir d'injures parce que, outre les 32 mesures réglementaires, son œuvre comporte une petite coda de 4 ou 8 mesures, et qu'il n'a pas répété la ligne mélodique à la main gauche, la chanson vous revient sous la forme d'un ozalid sur lequel, d'une plume ulcérée, vous tracez le fatidique B.A.T., bon à tirer, suivi de vos initiales, donnant ainsi votre caution à une œuvre sans rapport avec la géniale réalisation qu'elle était à l'origine, et qui, sous cet aspect fâcheux, va risquer maintenant de vous rapporter des sous 25 .

Censure? À l'évidence, le texte et la musique, que le compositeur et l'auteur signent de leur nom, contraints forcés, ne sont pas toujours ceux dont ils assument la paternité authentique; mais si, dans l'extrait ci-dessus, l'ironie de Vian travaille à défendre les droits du créateur, l'hyperbate finale est plus ambiguë : simple citation du discours de légitimation du censeur, qui prétexte le calcul de rentabilité pour assouvir et excuser sa pudibonderie et sa lâcheté? ou argument réaliste, validé nolens volens par Vian lui-même qui semble par ailleurs quelque peu relativiser la qualité de «la géniale réalisation» originelle? Sur cette dimension très matérielle de la genèse de la chanson, on peut se référer en outre aux témoignages qu'apportent les chansons des ACI : La Major (Les Wriggles), Signe-moi (Le Larron), et même la célèbre Ma chanson leur a pas plu (Renaud).

\section{Présentation des travaux : études de cas, entretiens, documents légendés}

Consacrée à Charles Gille (1820-1856), auteur, interprète et compositeur (malgréle peu d'airs de lui qui nous sont parvenus), l'étude de Romain Benini, érudite et passionnante, qui inaugure les études de cas, remonte au XIXe siècle; elle montre à quel point les difficultés de l'étude génétique d'une cuvre chansonnière reflètent le statut social de la chanson et sa "place dans les représentations collectives", surtout quand celle-ci est à la fois populaire (destinée au peuple) et politique (c'est-à-dire, en l'occurrence, subversive)26. Romain Benini pointe la rareté des manuscrits autographes, alors jugés peu intéressants à conserver, la diffusion essentiellement orale de la chanson, mais aussi la précarité matérielle des

25. Boris Vian, Euvres, En avant la zizique... et par ici les gros sous [1958, Le Livre contemporain], Paris, Fayard, vol. 12,2001 , p. 86 .

26. Comme le montre l'article de Stéphane Chaudier page 19, le discours génétique qui rapporte et explicite les étapes de la production d'une œuvre dépend du statut social qu' une collectivité confère à cette œuvre : on n'entreprend de raconter la genèse de Madame Bovary ou d'À la recherche du temps perdu qu'après et parce qu'une communauté quasi unanime de lettrés tient Flaubert et Proust pour des génies littéraires, dont les œuvres méritent d'être comprises de l'intérieur, en reconstituant soigneusement le processus qui les a fait naître. Rien de tel pour la chanson. Si l'on peut imaginer faire la genèse des textes de Brassens, Barbara, Ferré ou Gainsbourg, personne ne s'intéresse à celle des textes chantés par Mireille Mathieu, Gloria Lasso ou Vicky Léandros, lesquels sont à peine tenus pour des textes : de la chair émotionnelle à chanson, un simple tissu de stéréotypes sentimentaux censés mettre en valeur les voix étonnantes d'interprètes à succès. Mais tout cela peut changer; qui sait si, dans cinquante ans, Céline Dion et Dalida ne seront pas jugées beaucoup plus intéressantes que Brel et Barbara? On peut en douter; on ne saurait en jurer. 
auteurs (la Sacem n'est créée qu'en 1851) malgré l'extraordinaire popularité de Béranger (1780-1857) et la notoriété indéniable d'un Pierre Dupont (1821-1870) ou d'un Gustave Nadaud (1820-1893). À cela s'ajoute le fait que les textes imprimés dont on dispose sont souvent adultérés par la censure. Ainsi s'explique l'importance des textes manuscrits (autographes ou copies allographes), qui circulent sous le manteau, par rapport au volume des textes qui engagent la responsabilité d'un imprimeur. D'où la nécessité, une fois opéré le recensement des sources, d'examiner et de comparer les manuscrits disponibles : la ponctuation (souvent due à l'éditeur) mérite d'être confrontée à la partition, laquelle détermine en partie l'interprétation; enfin, outre de précieuses indications sur le caractère "évolutif» des chansons, les manuscrits autographes nous rendent sensibles aux corrections, stylistiques ou idéologiques, apportées par un éditeur comme Eugène Baillet, lesquelles ont le paradoxal mais réel avantage d'attirer notre attention sur les «lieux d'étrangeté ou de scandale textuels » dans la version originale.

Louis-Jean Calvet ${ }^{27}$ nous introduit dans l'atelier à chanson de Léo Ferré. Son article offre d'utiles jalons (chronologiques et biographiques) pour comprendre l'histoire d'un texte au titre quasi programmatique : La Mémoire et la mer, sorte de chanson en archipel, rhizome poétique ou matrice textuelle qui se dissémine dans six autres chansons et ne cesse de se transformer (1962-1982). De ce texte océanique, Louis-Jean Calvet présente une version inédite, dite de 1973, parce qu'elle lui a été donnée par l'artiste cette année-là; on pourrait sans doute comparer ce long poème au Bateau ivre ("Quand je me glisse dans le texte / La vague me prend tout mon sang ») tant l'analogie de la poésie et de la mer semble soustendre l'ensemble de la coulée verbale, parfois assez hermétique. Le mot «bateau » apparaît dans la chanson : "Un bateau, ça dépend comment / On l'arrime au port de justesse. » Comme dans le poème de Rimbaud, sans cesse se mêlent dans l'œuvre de Ferré Eros et Thanatos, initiative et dépossession, vigueur et mélancolie. Le poème s'achève "Devant la mer crépusculaire ». Mais du chaos naturel émerge quelque chose comme un chant: «ma lumière» sont bien les derniers mots de cette étonnante version de 1973.

Jean-Marc Quaranta, généticien, met sa spécialité au service de Brassens, d'emblée appréhendé comme "un polisseur de mots», un "auteur à genèse ». Si le matériau manque pour établir un véritable dossier génétique, il n'empêche que les différentes versions publiées d'une même chanson permettent déjà de dégager une ligne de force. Plus Brassens se corrige, plus il tend à effacer les traits quelque peu grossiers du chanteur grivois, amateur de scenarii burlesques, pour faire émerger une autre figure, gagnant en subtilité : celle d'un moi sensible, attentif à la complexité des relations humaines. Plaçant toute son étude sous l'égide d'un axiome de Ricardou pour qui l'écriture constitue non «le moyen d'expression d'une pensée déjà passée » mais "le moyen de production d'une pensée encore à venir», le travail de Jean-Marc Quaranta montre combien cette "pensée à venir» est, à propos de Brassens, fondamentalement une éthique : autant dire une représentation-construction de soi orientée vers la promotion d'un altruisme qui serait «l'évangile selon Brassens ».

27. Linguiste, Louis-Jean Calvet, né en 1942, s'est précocement et continûment intéressé à la chanson. Citons Cent Ans de chanson française (1972 pour la première édition; nombreuses rééditions depuis, dont la dernière, en poche, aux éditions l'Archipel); Georges Brassens, Payot, 1993 ; Léo Ferré, Flammarion, 2003 ; Chanson, la bande-son de notre histoire, l'Archipel, 2013 et une biographie de Moustaki, en 2014, chez le même éditeur. 
Suivent les articles d'Ursula Mathis-Moser et Olivier Migliore. Tous les deux s'attachent à décrire la concurrence entre deux textes et deux interprétations de la même chansonl'identité de l'œuvre étant consacrée par le titre et la quasi-similitude des paroles et de la partition. La première analyse Le Déserteur (Vian vs Mouloudji), Olivier Migliore Ma liberté (Moustaki vs Reggiani). Dans les deux cas, un auteur-compositeur (Vian et Moustaki) se voit librement dépossédé de sa maîtrise sur une ouvre dont le sens glisse et lui échappe dès lors qu'elle est interprétée par un rival (fût-il par ailleurs un ami ou un bon camarade). Le texte originel / original de Vian, très audacieusement antimilitariste, est recouvert par la récriture (souvent jugée, et à juste raison, affadissante) qu'en donne Mouloudji, auquel Vian finit pourtant par emboîter le pas, en ce qui concerne l'éthique du personnage : ce palimpseste chansonnier est un cas d'école. Le travail d'Ursula Mathis-Moser sensibilise à l'historicité de la chanson : si le genre "chanson » a été brillamment défini par Stéphane Hirschi comme un air fixé par des paroles, que reste-t-il d'une chanson quand les paroles sont elles-mêmes défigées par... l'air du temps? Le temps qui passe, comme le furet, remodèle les identités chansonnières qu'on aurait cru les plus stables, les plus pérennes, puisqu'on les avait et savait inscrites au panthéon de nos mémoires et de nos admirations collectives.

Olivier Migliore se livre à une démonstration très similaire, dans une perspective musicologique cette fois. Moustaki écrit Ma liberté; se conformant au programme éthique de la chanson, Reggiani s'approprie l'œuvre... Involontairement, il trahit les intentions restées implicites de Moustaki, et dont ce dernier n'avait peut-être pas conscience avant d'entendre le travail de déformation créatrice de Reggiani. En réinterprétant la chanson, Moustaki révèle l'impensé d'une intention, qui n'existe peut-être pas indépendamment d'une prise de risque interprétatif. Vertige cognitif... C'est l'interprétation « seconde » de Moustaki qui se révélerait la plus proche de l'œuvre "originelle»-dépositaire et garante du sens. À moins de convenir que le sens de Ma liberté n'était pas fixé a priori dans le marbre d'une introuvable intention d'auteur à laquelle se conformerait l'interprète; il s'actualise dans des interprétations successives et donc sujettes au passage du temps; la seconde, celle de l'auteur-compositeur devenu interprète de lui-même (Moustaki) vaudra la première, celle du «simple» chanteur (Reggiani) et aucune des deux ne peut prétendre être la version originale, la source des variations ultérieures.

Joël July conclut la série des études par un paradoxe : comment des chansons écrites par une auteure aussi indiscutablement reconnue que Barbara pourraient-elles être qualifiées de textes sans... autorité? La réponse est simple. Barbara chante Barbara (et elle en était très fière) mais elle ne chante pas que Barbara; disons donc qu'elle est chanteuse (et se perçoit comme telle) autant que poète (mais elle-même refuse le mot). En tant que chanteuse, elle ne peut interrompre sa performance vocale et revendique haut et fort l'organique coulée d'une interprétation; mais c'est aussi parce qu'elle est chanteuse qu'elle déforme incessamment, au gré de leurs mises en voix successives, des textes qui cessent d'être des unités stables, figées-fixées par le geste auctorial et le prestige de l'imprimé. C'est en chanteuse, au piano, seule ou sur scène, en tournée plus souvent qu'à la maison, qu'elle élabore une chanson aux paroles toujours déjà variantes et variables; elle ajoute, retranche. Alors quel texte retenir? Quel enregistrement? Par ailleurs la voix joue avec la métrique, allongeant ou accourcissant le vers. Peu importe le numérisme, le contenu du vers : la voix décide et les musiciens suivent. Au terme de ce parcours, on serait donc presque tentés de reprendre (et d'adapter à la 
chanson) le fameux précepte de la phénoménologie : "Autant d'apparaître, autant d'être 28 » et dire avec audace : "Autant d'interprétations, autant de chansons ».

La section Entretiens prolonge et enrichit les études : on y entend un métadiscours (à la première personne) sur l'expérience créatrice. Dans l'échange très nourri avec Gaspard Turin, Bertrand Belin aborde des thèmes qui débordent le cadre de la problématique génétique; tout au long de ses propos, Bertrand Belin souligne la fluidité du processus génétique en chanson. Il avance trois arguments tirés de sa pratique qu'on peut recevoir comme autant de preuves empiriques. D'une part, le guitariste qu'il est ne compose pas la chanson à la manière d'un poème dont les strates successives seraient déposées sur un support. Il «marmonne» des mots dans un micro, archive en sa mémoire une phrase venue lors d'un concert et à partir de laquelle il improvise : "Et quand j'ai eu la musique de la chanson dans les oreilles, j'avais, dans mon vestiaire de choses faites, cette phrase, que j'avais prononcée sur scène mais qui n'était écrite nulle part. »Certes, il lui arrive aussi de jeter sur une page un mot ou deux, mais ce sont "des choses qui ne se présentent pas comme des chansons ». C'est au moment de la composition musicale que l'émulsion se fait : «Je prends mes petites notes et je les regarde, comme ça, n'importe comment, pendant que la musique se déroule. Ça prend forme ainsi. » Ensuite, la genèse de la chanson ne se déroule nullement dans le "for intérieur", mythique et clos, d'une création ex nihilo. En y regardant de plus près, ce fameux for est bien, conformément à ce que dit l'étymologie, un véritable forum polyphonique, un carrefour culturel : les musiques et les chansons aimées y affluent, mais aussi les souvenirs de films ou de tableaux marquants; et c'est en liaison avec ces formes préexistantes, imposant une atmosphère, que la chanson advient : elle procède d'un dialogue fait du double désir de continuer et de renouveler les auvres nourricières. Enfin, à l'instar des artistes les plus exigeants, comme Dylan, l'enregistrement ne fige pas l'auvre de Belin; il ne s'agit pas pour lui de reproduire ce qui a été archivé par le studio, pérennisé par le disque, mais bien de faire évoluer l'œuvre au gré des circonstances, au risque parfois de la rendre méconnaissable. Toutefois, à reporter l'exigence de l'artiste sur son public, on peut imaginer que les fans y trouveront leur compte.

Le point de vue de Philippe Djian est différent, puisque ce dernier, romancier reconnu, n'est qu'un parolier occasionnel, travaillant pour Stephan Eicher. L'entretien qu'il accorde à Élodie Burle-Errecade s'ouvre sur la question des relations entre roman et chanson. Ces deux modes d'expression s'efforcent de capter un air du temps; ce sont deux «traductions mélodiques » du monde où nous vivons. Inscrites dans la chair de la fiction romanesque, les chansons servent à renforcer le lien avec le lecteur, surtout quand ce dernier, grâce au roman - et le cas arrive, affirme Djian -, découvre de nouvelles chansons. Comme la météo dont les récits de Djian font grand usage, la chanson crée un effet de réel. Elle ancre la scène dans une "atmosphère». Les chansons sont partout dans l'espace social : elles arrivent à nous sans qu'il faille faire d'effort pour venir à elles; à cause des vers, du rythme, elles s'impriment par fragments dans nos têtes - et cela suffit à assurer leur pérennité : "Parfois on change un mot, une phrase / Et quand on est à court d'idées / On fait la la la la la la», disait déjà Trenet, dans L'Âme des poètes (1951).

28. La formule de Husserl soviel Schein, soviel Sein se trouve dans les Méditations cartésiennes, trad. fr. E. Levinas et G. Peiffer, Paris, Vrin, rééd. 1986, § 46, p. 169, dont le texte exact est : «tant d'apparence, tant d'être». 
Même s'il ne monte pas en scène, Djian se sent obligé, quoique piètre musicien, de prendre sa guitare en main pour faire surgir un rythme premier. De cette émulsion originelle (texte et notes), il ne reste souvent rien puisque Djian n'est pas mélodiste; cette phase s'avère pourtant indispensable, car une chanson ne s'écrit pas dans le vide; elle est composée pour un chanteur, qui a ses prédilections ou ses difficultés articulatoires; elle est offerte à un musicien qui devra trouver la mélodie juste pour accompagner le texte et «défendre "la chanson face à un public. Il se peut que, malgré une complicité éprouvée, presque magique, entre le parolier et l'interprète, il y ait entre eux des malentendus, des contresens: ce que l'un avait écrit avec telle intention, l'autre le comprend différemment et fait entendre tout autre chose.

Le texte de chanson n'est donc pas sanctuarisé : il faut, précise Djian, "ne pas prendre ça pour de la poésie, dont on ne peut même pas toucher une virgule, ni enlever un mot». Au contraire : le parolier doit accepter que ses paroles lui échappent, d'autant plus, estime-t-il, qu'elles ne font pas l'essentiel de la chanson; elles sont le tremplin d'une ouvre imprévisible, laquelle appartient au chanteur qui s'en est emparé. "C'est un drôle d'objet, la chanson : ce n'est jamais moi tout seul», reconnaît Philippe Djian. Et c'est bien la nature de cet «objet-chanson» qui rend si particulier ou si problématique l'examen de sa genèse. Le parolier n'est pas un «auteur» comme les autres, en ce sens que l'interprète peut sans cesse transformer son texte, au cours de la mise en musique ou même sur scène : "À chaque fois, c'est une nouvelle composition qui se met en place », conclut Djian. "Le principal c'est que ça fonctionne, que ça marche, voilà. » Et c'est pourquoi, en l'écoutant à la radio, en l'entendant chantée et reprise en concert, le parolier ne reconnaît parfois plus sa chanson, son texte. Et c'est de bon augure...

Comment mieux finir que par des images? Louis-Jean Calvet offre et commente le texte de Love en faisant apparaître une correction joliment stylistique (la répétition du mot «tête», dans la troisième strophe, renforce l'effet de symétrie d'un vers à l'autre) et une variante importante. Dans la série d'images qui suivent, on découvre comment le livre qui inspire Bertrand Belin pour deux de ses chansons (Claude Simon, Archipel et Nord) se mue en carnet de notes - comme si la chanson s'élaborait dans les marges du texte, dans la proximité la plus étroite avec sa source littéraire. Enfin, on revient à Georges Brassens avec le manuscrit de La Religieuse, et ses nombreuses versions. 